\title{
SOME REMARKS ON FLOCKS
}

\author{
LAURA BADER, CHRISTINE M. O'KEEFE and TIM PENTTILA
}

(Received 14 March 2002; revised 3 March 2003)

Communicated by B. D. McKay

\begin{abstract}
New proofs are given of the fundamental results of Bader, Lunardon and Thas relating flocks of the quadratic cone in $\operatorname{PG}(3, q), q$ odd, and BLT-sets of $\mathrm{Q}(4, q)$. We also show that there is a unique BLT-set of $\mathrm{H}(3,9)$. The model of Penttila for $\mathrm{Q}(4, q), q$ odd, is extended to $\mathrm{Q}(2 m, q)$ to construct partial flocks of size $q m / 2+m / 2-1$ of the cone $\mathscr{K}$ in $\mathrm{PG}(2 m-1, q)$ with vertex a point and base $\mathrm{Q}(2 m-2, q)$, where $q$ is congruent to 1 or 3 modulo 8 and $m$ is even. These partial flocks are larger than the largest previously known for $m>2$. Also, the example of O'Keefe and Thas of a partial flock of $\mathscr{K}$ in $\mathrm{PG}(5,3)$ of size 6 is generalised to a partial flock of the cone $\mathscr{K}$ of $\mathrm{PG}(2 p n-1, p)$ of size $2 p n$, for any prime $p$ congruent to 1 or 3 modulo 8, with the corresponding partial BLT-set of $\mathrm{Q}(2 p n, p)$ admitting the symmetric group of degree $2 p n+1$.
\end{abstract}

2000 Mathematics subject classification: primary 51E12; secondary 51A40, 51 A50, 51E20, $05 B 25$. Keywords and phrases: flock, generalised quadrangle, BLT-set, parabolic quadric, ovoid.

\section{Introduction}

The central role of BLT-sets in the rapidly expanding area of the study of flocks of Miquelian Laguerre planes of odd order and the related elation generalised quadrangles and translation planes is exemplified by Knarr's construction of the quadrangle from the BLT-set. Here we consider a number of generalisations of BLT-sets of Q $(4, q), q$ odd: to BLT-sets of $\mathrm{H}\left(3, q^{2}\right), q$ odd, in Section 5; to BLT-sets of $\mathrm{T}_{2}^{*}(\mathscr{H})$, in Section 6; to BLT-sets of finite generalised quadrangles in general, in Section 2; to BLT-sets of the polar spaces $\mathrm{Q}(2 n, q), q$ odd, of rank $n>2$, in Section 7. The material in Section 2 is related to work of De Soete and Thas [4], predating the introduction of BLT-sets of $\mathrm{Q}(4, q)$ in Bader, Lunardon and Thas [2] by six years. While we note the advances of Shult and Thas [14] in Section 3, we restrict ourselves to BLT-sets of points, rather than sets of subspaces with the BLT-property.

(C) 2004 Australian Mathematical Society 1446-7887/04 \$A2.00+0.00 
In Section 4, we give new proofs of the fundamental results of Bader, Lunardon and Thas [2] relating flocks of the quadratic cone of $\mathrm{PG}(3, q), q$ odd, and BLT-sets of $\mathrm{Q}(4, q)$. In Section 5, we show that there is a unique BLT-set of $\mathrm{H}(3,9)$. In Section 7 , partial flocks of $\mathrm{Q}(2 m, q)$ larger than those previously known are constructed, both by extending the model of Penttila [12] of $\mathrm{Q}(4, q), q$ odd, to $\mathrm{Q}(4 n, q)$ to obtain partial flocks of size $q n+n-1$ of the cone $\mathscr{K}$ in $\operatorname{PG}(4 n-1, q)$ with vertex a point and base $\mathrm{Q}(4 n-2, q)$, where $q$ is congruent 1 or 3 modulo 8 , and by generalising the example of O'Keefe and Thas [9] to a partial flock of size $2 p n$ in $\operatorname{PG}(2 p n-1, p)$ for any prime $p$ congruent 1 or 3 modulo 8 .

The paper is structured as a sequence of remarks. For reasons of space, we refer the reader to $[2,1,8,9,10,16,17]$ for background material and definitions.

\section{Definition, combinatorics and non-existence results}

Let $\mathscr{S}=(\mathscr{P}, \mathscr{L}, l)$ be a generalised quadrangle of $\operatorname{order}(s, t)$, where $s, t>1$. A partial BLT-set of $\mathscr{S}$ is a set $\mathscr{B}$ of $b \geq 3$ points such that for all distinct $P, Q, R \in \mathscr{B}$ we have $\{P, Q, R\}^{\perp}=\emptyset$. Equivalently, no point of $\mathscr{S}$ is collinear with more than two points of $\mathscr{B}$.

LEMMA 2.1. Let $\mathscr{B}$ be a partial BLT-set in the generalised quadrangle $\mathscr{S}$ of order $(s, t)$. Then no two points of $\mathscr{B}$ are collinear.

Proof. Suppose that $P, Q \in \mathscr{B}$ are collinear, $P \neq Q$. If $R \in \mathscr{B}$ is on the line $P Q, P \neq R \neq Q$, then $R \in\{P, Q, R\}^{\perp}$; a contradiction. If $R \in \mathscr{B}$ is not on the line $P Q$ then there is a unique point $X \in P Q$ collinear with $R$, whence $X \in\{P, Q, R\}^{\perp}$; a contradiction.

It is immediate that $\mathscr{B}$ is a partial BLT-set if and only if it is a set of $b \geq 3$ points, each three of which form an acentric triad (equivalently, each three of which form a partial BLT-set).

LEMMA 2.2. Let $\mathscr{B}$ be a partial BLT-set in the generalised quadrangle $\mathscr{S}$ of order $(s, t)$. Then $b=|\mathscr{B}| \leq s+1$.

Proof. For $i=0,1,2$, let $t_{i}$ be the number of points of $\mathscr{P} \backslash \mathscr{B}$ collinear with exactly $i$ points of $\mathscr{B}$. Counting in two ways the points of $\mathscr{P} \backslash \mathscr{B}$, the pairs $(P, T)$ where $P \in \mathscr{B}$ and $T \in \mathscr{P} \backslash \mathscr{B}$ are collinear and the ordered triples $(P, Q, T)$ where $P, Q \in \mathscr{B}, P \neq Q$, and $T \in \mathscr{P} \backslash \mathscr{B}$ are such that each of $P$ and $Q$ is collinear with $T$, we obtain:

$$
t_{0}+t_{1}+t_{2}=(s+1)(s t+1)-b,
$$




$$
\begin{aligned}
t_{1}+2 t_{2} & =b(t+1) s, \\
2 t_{2} & =b(b-1)(t+1) .
\end{aligned}
$$

Since $t_{1}=b(t+1)(s-b+1) \geq 0$, the result follows.

Let $\mathscr{S}$ be a generalised quadrangle of order $(s, t)$, where $s, t>1$. A BLT-set in $\mathscr{S}$ is a partial BLT-set in $\mathscr{S}$ of size (maximal) $s+1$. It is immediate from the proof of Lemma 2.2 that if $\mathscr{B}$ is a BLT-set in $\mathscr{S}$ then for each point $X \in \mathscr{P} \backslash \mathscr{B}$ we have $\left|X^{\perp} \cap \mathscr{B}\right| \in\{0,2\}$, and that $\mathscr{S}$ admits $t_{0}=s(s+1)(t-1) / 2$ points of $\mathscr{P} \backslash \mathscr{B}$ collinear with no point of $\mathscr{B}$ and $t_{2}=s(s+1)(t+1) / 2$ points collinear with exactly two points of $\mathscr{B}$.

Some of these results have already been obtained by De Soete and Thas [4], who define a $(0,2)$-set in $\mathscr{S}$ to be a non-empty set $\mathscr{B}$ of pairwise non-collinear points such that $\left|X^{\perp} \cap \mathscr{B}\right| \in\{0,2\}$ for all $X \in \mathscr{P} \backslash \mathscr{B}$. They prove that if $\mathscr{S}$ admits a $(0,2)$-set $\mathscr{B}$ then $|\mathscr{B}|=s+1$; so the concepts of BLT-set and $(0,2)$-set in a generalised quadrangle of $\operatorname{order}(s, t), s, t>1$, coincide.

LEMMA 2.3 (De Soete and Thas [4]). Let $\mathscr{S}$ be a generalised quadrangle of order $(s, t)$, where $s, t>1$. If there exists a BLT-set in $\mathscr{S}$ then $s$ is odd.

ProOF. Let $\mathscr{B}$ be a BLT-set of $\mathscr{S}$. The number of lines of $\mathscr{S}$ not meeting $\mathscr{B}$ is $(t+1)(s t+1)-(s+1)(t+1)=s(t+1)(t-1)>0$; let $\ell$ be such a line. For each point $P \in \mathscr{B}$, there exists a unique point $T \in \ell$ collinear with $P$, and a unique point $P^{\prime} \in \mathscr{B} \backslash\{P\}$ collinear with $T$. It is immediate that $|\mathscr{B}|=s+1$ is even, so $s$ is odd.

LEMMA 2.4. Let $\mathscr{S}$ be a generalised quadrangle of order $s$. For any regular pair $(x, y)$, there is no partial BLT-set containing both $x$ and $y$.

PROOF. From $[10,1.3 .6$ (ii)] it follows that any triad $(x, y, z)$ has a centre.

COROLLARY 2.5 (De Soete and Thas [4]). The generalised quadrangle W(q) has io partial BLT-set.

PROOF. Every pair in $\mathrm{W}(q)$ is regular.

COROLLARY 2.6. Let $\mathscr{B}$ be a partial BLT-set in $\mathrm{T}_{2}(\mathscr{O})$ for $\mathscr{O}$ an oval (which is not i conic) in $\mathrm{PG}(2, q), q$ even. Then $(\infty) \notin \mathscr{B}$ and $\mathscr{B}$ has at most one point of type (ii).

Proof. The point $(\infty)$ is regular. Any pair of points of type (ii) is collinear with he regular point $(\infty)$ and therefore the pair is regular. 
The known (non-isomorphic) examples of generalised quadrangle of order $s$ all have $s=q$, a prime power. They are: $\mathrm{W}(q), \mathrm{Q}(4, q)$ for $q$ odd and $\mathrm{T}_{2}(\mathscr{O})$ (and its dual) for $\theta$ an oval (which is not a conic) in $\operatorname{PG}(2, q), q$ even. The only example which could admit a BLT-set is $\mathrm{Q}(4, q)$ for $q$ odd. In fact there are many interesting examples of such BLT-sets which we study further in Section 4 . The example $\mathrm{T}_{2}(\mathscr{O})$ for $\mathscr{O}$ an oval (which is not a conic) in $\operatorname{PG}(2, q), q$ even, does not admit a BLT-set (by Lemma 2.3), but may admit partial BLT-sets.

LEMMA 2.7. Any generalised quadrangle of order $\left(s, s^{2}\right), s>1$ has no partial BLT-set.

ProOF. By a result of Bose and Shrikhande (see $[10,1.2 .4]$ ), every triad in a generalised quadrangle of order $\left(s, s^{2}\right), s>1$, has exactly $s+1$ centres.

The next result is due to Thas (see Knarr [8]) for (complete) BLT-sets.

LEMMA 2.8. $\mathrm{H}\left(4, q^{2}\right)$ has no partial BLT-set.

PROOF. The polar space of the subspace spanned by three points of $\mathrm{H}\left(4, q^{2}\right)$ is either a line or plane, so it meets the variety $\mathrm{H}\left(4, q^{2}\right)$. Therefore, every triad has a centre.

\section{BLT-sets and related structures}

The next construction, due to Knarr [8], shows that to a dual BLT-set in W $(q), q$ odd, there corresponds a generalised quadrangle of order $\left(q^{2}, q\right)$.

THEOREM 3.1 (Knarr [8]). Let $P$ be a point of $\mathrm{W}(5, q)$, let $\mathrm{W}(q)=P^{\perp} / P$ and let $\mathscr{B}$ be a BLT-set in $\mathrm{W}(q)$. So $\mathscr{P}=\{\langle P, \ell\rangle: \ell \in \mathscr{B}\}$ is a set of $q+1$ planes of $\mathrm{W}(5, q)$ on $P$. Then the incidence structure with:

Points: (i) the point $P$, (ii) lines of $\mathrm{W}(5, q)$ on an element of $\mathscr{P}$ and not on $P$, (iii) points of $\mathrm{W}(5, q) \backslash P^{\perp}$;

Lines: (a) the elements of $\mathscr{P}$, (b) the planes of $\mathrm{W}(5, q)$ which meet $P^{\perp}$ in a line of the form (ii);

Incidence: natural;

is a generalised quadrangle of order $\left(q^{2}, q\right)$.

Payne and Thas in [11] have related the group of this generalised quadrangle with the subgroup of $\mathrm{P} \Gamma \mathrm{O}(5, q)$ stabilising the BLT-set. Also, Shult and Thas in [14] have vastly generalised this construction. 
Following is the well-known construction of the ovoid of $\mathrm{Q}^{+}(5, q)$ from a flock, but given directly from the BLT-set.

In $\mathrm{PG}(5, q), q$ odd, fix the quadric $\mathrm{Q}^{+}(5, q)$ and embed $\mathrm{Q}(4, q)$ in $\mathrm{Q}^{+}(5, q)$ as the section with a hyperplane $H$. Denote by $\perp$ the polarity defined by $\mathrm{Q}^{+}(5, q)$ and put $x=H^{\perp}$. Let $\mathscr{B}$ be a partial BLT-set of size $k+1$ of $\mathrm{Q}(4, q)$ and fix a point $p$ in $\mathscr{B}$. For each $p_{i} \in \mathscr{B}, p_{i} \neq p, i=1,2, \ldots, k$, let $C_{i}$ be the conic $\left(x, p, p_{i}\right) \cap \mathrm{Q}^{+}(5, q)$. The set $\mathscr{O}=C_{1} \cup C_{2} \cup \cdots \cup C_{k}$ is the union of $k$ conics with the common point $p$. Also, $\mathscr{O}$ is a partial ovoid because: no point of $\mathscr{O}$ different from $p$ is collinear with $p$ as they lie on some $C_{i}$; no two points on the same $C_{i}$ are collinear; take any $x_{i} \in C_{i}, x_{j} \in C_{j}$, $i \neq j$ with $x_{i} \neq p \neq x_{j}$; then $\left\langle x, x_{i}, x_{j}, p\right\rangle^{\perp}=\left\langle p, p_{i}, p_{j}\right\rangle^{\perp} \cap x^{\perp}$ is an external line because $\mathscr{B}$ is a partial BLT-set, hence $\left\langle x, x_{i}, x_{j}, p\right) \cap \mathrm{Q}^{+}(5, q)=\mathrm{Q}^{-}(3, q)$, thus $x_{i}$ and $x_{j}$ are not collinear.

Note this argument is an alternative way of looking at the so called Thas-Walker construction, and it works also for $\mathrm{Q}(2 n, q), q$ odd.

A BLT-set (of points) of $\mathrm{H}\left(3, q^{2}\right)$ has order $q^{2}+1$ and gives, by duality, a BLT-set $B$ of lines of $\mathrm{Q}^{-}(5, q)$, that is a set of mutually skew lines of $\mathrm{Q}^{-}(5, q)$ such that no three of them have a common tranversal on $\mathrm{Q}^{-}(5, q)$. Shult and Thas [14] prove that a translation generalised quadrangle of order $q^{2}$ arises.

THEOREM 3.2 (Shult and Thas [14]). Fix a point $P$ of $\mathrm{Q}^{-}(7, q)$. Let $\mathrm{Q}^{-}(5, q)=$ $P^{\downarrow} / P$ and let $B$ be a BLT-set of lines in $\mathrm{Q}^{-}(5, q)$. So $\mathscr{P}=\{\langle P, \ell\rangle: \ell \in \mathscr{B}\}$ is a set of $q^{2}+1$ planes of $\mathrm{Q}^{-}(7, q)$ on $P$. Then the incidence structure with:

Points: (i) the point $P$, (ii) lines of $\mathrm{Q}^{-}(7, q)$ on an element of $\mathscr{P}$ and not on $P$, (iii) points of $\mathrm{Q}^{-}(7, q) \backslash P^{\perp}$;

Lines: (a) the elements of $\mathscr{P}$, (b) the planes of $\mathrm{Q}^{-}(7, q)$ which meet $P^{\perp}$ in a line of type (ii);

Incidence: natural;

is a translation generalised quadrangle of order $q^{2}$.

Furthermore, we briefly recall the following construction. For more details, see 10] and [1]. An egg $\mathrm{O}(2,2, q)$, say $\mathscr{E}$, is a partial spread of lines in $\operatorname{PG}(5, q)$ such hat:

(1) $\mathscr{E}$ contains $q^{2}+1$ elements;

(2) every three elements of $\mathscr{E}$ generate $\mathrm{PG}(5, q)$;

(3) each element $X$ of $\mathscr{E}$ is contained in a 3-dimensional subspace $T_{X}$ having no soint in common with any element of $\mathscr{E}$ different from $X$. The subspace $T_{X}$ is unique Ind is called the tangent space to $\mathscr{E}$ at $X$.

Embed $\operatorname{PG}(5, q)$ in $\operatorname{PG}(6, q)$ as a hyperplane, and define an incidence structure $\Gamma(\mathscr{E})$ as follows. Points are: (i) a new symbol $(\infty)$, (ii) the 4-dimensional subspaces $\checkmark$ of $\operatorname{PG}(6, q)$ for which $X \cap \operatorname{PG}(5, q)$ is a tangent space to $\mathscr{E}$, and (iii) the points of 
PG $(6, q) \backslash \mathrm{PG}(5, q)$. Lines are: (a) the elements of $\mathscr{E}$, and (b) the planes of PG(6,q) which are not contained in $\mathrm{PG}(5, q)$ and meet $\mathrm{PG}(5, q)$ in elements of $\mathscr{E}$. Incidence is defined as follows. The point $(\infty)$ is incident with all lines of type (a) and no line of type (b). A point of type (ii) is incident with all lines of type (b) contained in it and with the unique element of $\mathscr{E}$ incident with it. A point of type (iii) is incident only with lines of type (b); here the incidence is that of $\operatorname{PG}(6, q)$.

Payne and Thas $[10,8.7 .1]$ proved that $T(\mathscr{E})$ is a translation generalized quadrangle of order $q^{2}$ with base point $(\infty)$.

\section{BLT-sets in $Q(4, q)$ and flocks of the quadratic cone in $\operatorname{PG}(3, q)$}

Let $V$ be a 5-dimensional vector space over $\mathrm{GF}(q), q$ odd, let $Q$ be a non-degenerate quadratic form on $V$ and let $f$ be the bilinear polar form of $Q$, that is,

$$
f(x, y)=Q(x+y)-Q(x)-Q(y) \quad \text { for } x, y \in V .
$$

Let $\perp$ denote the polarity determined by $f$. Let $E=\left\{e_{1}, \ldots, e_{5}\right\}$ be a basis for $V$ over $\mathrm{GF}(q)$ and let $d(f, E)$ be the determinant of the matrix $\left[f\left(e_{i}, e_{j}\right)\right]_{i, j=1, \ldots, 5}$. If $E^{\prime}$ is any basis for $V$ over $\operatorname{GF}(q)$ then $d\left(f, E^{\prime}\right)=d(f, E) a^{2}$ where $a$ is the determinant of the transition matrix between the two bases. It follows that the set $d(f, E) F^{2}$ (where $\left.F^{2}=\left\{a^{2}: a \in \mathrm{GF}(q) \backslash\{0\}\right\}\right)$ is an invariant, called the discriminant, of the quadratic form $Q$ (in other words, the property that $d(f, E)$ is a square in $\operatorname{GF}(q)$ or not is an invariant of $Q$ ). In the following we will use $\Delta$ to denote an arbitrary element of $d(f, E) F^{2}$, since we are usually only interested in whether this element is a square or not.

LEMMA 4.1. Let $q$ be odd. Suppose $x, y, z$ are linearly independent vectors of $V$ such that $\langle x\rangle,\langle y\rangle,\langle z\rangle$ are singular points with respect to $Q$. Then $\langle x, y, z\rangle^{\perp}$ is anisotropic if and only if $-2 f(x, y) f(y, z) f(z, x) / \Delta$ is a non-square in $\operatorname{GF}(q)$.

ProOf. Let $W=\langle x, y, z\rangle$ and suppose that $W^{\perp}$ is anisotropic. Then $\operatorname{dim} W^{\perp}=2$, $\operatorname{dim} W=3$ and $V=W \perp W^{\perp}$, where $W^{\perp}=\langle u, v\rangle$ for some $u, v \in V$. Thus $E=\{x, y, z, u, v\}$ is a basis for $V$ over $\operatorname{GF}(q)$ and

$$
-\left|\begin{array}{ll}
f(u, u) & f(u, v) \\
f(v, u) & f(v, v)
\end{array}\right|
$$

is a non-square in $\operatorname{GF}(q)$; but it also equals

$$
\frac{-d(f, E)}{2 f(x, y) f(y, z) f(z, x)}
$$


and the result follows.

Conversely, suppose that $-2 f(x, y) f(y, z) f(z, x) / \Delta$ is a non-square in $\operatorname{GF}(q)$. Let $W=\langle x, y, z\rangle$. Then

$$
\left|\begin{array}{lll}
f(x, x) & f(x, y) & f(x, z) \\
f(y, x) & f(y, y) & f(y, z) \\
f(z, x) & f(z, y) & f(z, z)
\end{array}\right|=2 f(x, y) f(y, z) f(z, x)
$$

is not zero, so $W$ is non-degenerate and $V=W \perp W^{\perp}$. Let $W^{\perp}=\langle u, v\rangle$, then $E=\{x, y, z, u, v\}$ is a basis for $V$ over $\operatorname{GF}(q)$ and

$$
-\left|\begin{array}{rr}
-f(u, u) & f(u, v) \\
f(v, u) & f(v, v)
\end{array}\right|=\frac{-d(f, E)}{2 f(x, y) f(y, z) f(z, x)}
$$

is a non-square in $\operatorname{GF}(q)$; so $W^{\perp}$ is anisotropic.

COROLLARY 4.2. Let $q$ be odd. Let $\mathscr{B}$ be a set of $b \geq 3$ points of $Q(4, q)$ and let $f$ be the bilinear form corresponding to the quadratic form underlying $\mathrm{Q}(4, q)$. Then $\mathscr{B}$ is a partial BLT-set if and only iffor all $x, y, z \in \mathscr{B}$ we have: $-2 f(x, y) f(y, z) f(z, x) / \Delta$ is a non-square in $\mathrm{GF}(q)$.

LEMMA 4.3. Let $q$ be odd. Let $\mathscr{B}=\{x, y, z, w\}$ be a set of four points of $\mathrm{Q}(4, q)$ such that $\{x, y, z\},\{x, z, w\}$ and $\{x, w, y\}$ are partial BLT-sets. Then $\mathscr{B}$ is a partial BLT-set.

Proof. It suffices to check that $\{y, z, w\}$ is a partial BLT-set. Now by Corollary 4.2 we know that $-2 f(x, y) f(y, z) f(z, x) / \Delta,-2 f(x, z) f(z, w) f(w, x) / \Delta$ and $-2 f(x, w) f(w, y) f(y, x) / \Delta$ are all non-squares, hence their product

$$
\left(\frac{-2 f(x, y) f(x, z) f(x, w)}{\Delta}\right)^{2} \frac{-2 f(y, z) f(z, w) f(w, y)}{\Delta}
$$

is a non-square and the result follows.

This proof was inspired by Seidel [13], where a very similar argument is used to construct a 2-graph.

As an immediate corollary we obtain the following slight generalisation, originally due to Johnson [6].

COROLlaRY 4.4 (Johnson [6]). Let $q$ be odd. Let $\mathscr{B}$ be a set of $b \geq 3$ points of $\mathrm{Q}(4, q)$. Suppose there exists $x \in \mathscr{B}$ such that $\{x, y, z\}$ is a partial BLT-set for all $y, z \in \mathscr{B} \backslash\{x\}$. Then $\mathscr{B}$ is a partial BLT-set. 
Bader, Lunardon and Thas [2, Remark 1] linked BLT-sets in Q(4,q) with flocks of quadratic cones in $\mathrm{PG}(3, q)$.

THEOREM 4.5. Let $q$ be odd. To each point of a BLT-set in $\mathrm{Q}(4, q)$ there corresponds a flock of a quadratic cone in $\mathrm{PG}(3, q)$. Conversely, to a flock of a quadratic cone in $\mathrm{PG}(3, q)$ there corresponds $a \mathrm{BLT}$-set with a distinguished point.

Proof. Let $\mathscr{B}$ be a BLT-set in $\mathrm{Q}(4, q)$ and let $P \in \mathscr{B}$. Then $P^{\perp} \cap \mathscr{Q}(4, q)$ is a quadratic cone in the 3-dimensional space spanned by $P^{\perp}$, and we consider the set of $q$ planes $\mathscr{F}=\left\{P^{\perp} \cap Q^{\perp}: Q \in \mathscr{B} \backslash\{P\}\right\}$. For $Q, R \in \mathscr{B} \backslash\{P\}, Q \neq R$, we have $\left(P^{\perp} \cap Q^{\perp}\right) \cap\left(P^{\perp} \cap R^{\perp}\right)=\emptyset$; so no two planes of $\mathscr{F}$ contain a common point of $\mathrm{Q}(4, q)$ and $\mathscr{F}$ is a flock of $P^{\perp} \cap \mathrm{Q}(4, q)$. Conversely, let $\mathscr{F}$ be a flock of a quadratic cone $\mathscr{K}$ with vertex $P$ in $\mathrm{PG}(3, q)$. Embed $\mathscr{K}$ in a non-singular quadric $\mathrm{Q}(4, q)$, so that $\mathscr{K}=P^{\perp} \cap \mathrm{Q}(4, q)$. For $\pi \in \mathscr{F}, \pi^{\perp}$ is a line on $P$ which meets $\mathrm{Q}(4, q)$ in a further point and we let

$$
\mathscr{B}=\{P\} \cup\left\{\left(\pi^{\perp} \cap Q(4, q)\right) \backslash\{P\}: \pi \in \mathscr{F}\right\} .
$$

For $Q, R \in \mathscr{B} \backslash\{P\}, Q \neq R$, we have

$$
P^{\perp} \cap Q^{\perp} \cap R^{\perp}=P^{\perp} \cap(P Q)^{\perp} \cap(P R)^{\perp}=\emptyset .
$$

By Corollary $4.4, \mathscr{B}$ is a BLT-set.

COROLLARY 4.6. Two points of a $\mathrm{BLT}$-set in $\mathrm{Q}(4, q)$ give rise to projectively equivalent flocks of a quadratic cone in $\mathrm{PG}(3, q)$ if and only if they are in the same orbit of the stabiliser of the $\mathrm{BLT}$-set in the stabiliser $\mathrm{P} \Gamma \mathrm{O}(5, q)$ of $\mathrm{Q}(4, q)$.

\section{BLT-sets in $\mathrm{H}\left(3, q^{2}\right)$}

Apart from a brief mention in Knarr [8], BLT-sets in $\mathrm{H}\left(3, q^{2}\right)$ have received little attention to date. Knarr [8] attributes the following examples to Thas.

THEOREM 5.1. Let $q$ be odd. Then, $\mathrm{Q}^{-}(3, q)$ is a BLT-set of $\mathrm{H}\left(3, q^{2}\right)$.

Proof. Let $\mathrm{Q}^{-}(3, q)$ have equation $y^{T} A y=0$, with $y$ homogeneous projective coordinates in $\operatorname{PG}(3, q)$. Note that $A$ is a symmetric matrix with entries in $\operatorname{GF}(q)$. Embed $\mathrm{PG}(3, q)$ in $\mathrm{PG}\left(3, q^{2}\right)$. Denote by $\pi$ the polarity defined by $\mathrm{Q}^{-}(3, q)$ and by $\sigma$ the Baer involution of $\operatorname{PG}\left(3, q^{2}\right)$ defined by the automorphism $x \in \mathrm{GF}\left(q^{2}\right) \mapsto$ $x^{q} \in \mathrm{GF}\left(q^{2}\right)$. The map $\pi \sigma$ is a unitary polarity of $\operatorname{PG}\left(3, q^{2}\right)$, thus it defines a variety $H\left(3, q^{2}\right)$ (with equation $y^{T} A y^{\sigma}=0$ ) with the $Q^{-}(3, q)$ embedded. Given any 
three distinct points $x, y, z$ on $\mathrm{Q}^{-}(3, q),\langle x, y, z\rangle$ is a plane, its polar point $v$ (with respect to the polarity $\pi \sigma)$ is in $\operatorname{PG}(3, q)$ because $x, y$ and $z$ so are, and $v$ is not on $\mathrm{H}\left(3, q^{2}\right)$ because $\mathrm{Q}^{-}(3, q)=\mathrm{H}\left(3, q^{2}\right) \cap \mathrm{PG}(3, q)$. Hence, any triad on $\mathrm{Q}^{-}(3, q)$ is acentric.

We only contribute a classification in the smallest case.

THEOREM 5.2. The unique BLT-set of $\mathrm{H}(3,9)$ is $\mathrm{Q}^{-}(3,3)$.

PROOF. Recall that for $s$ odd (see Payne and Thas [10]) generalised quadrangles of order $s$ with an antiregular point are equivalent to Laguerre planes LP( $s)$ of order $s$ (see Delandtsheer [5]), and the base point of a translation generalised quadrangle of order $s$ ( $s$ odd) is antiregular. By Theorem 5.1, the elliptic quadric $\mathrm{Q}^{-}(3, q)$ is a BLT-set of $\mathrm{H}\left(3, q^{2}\right)$, and using Theorem 3.2 a translation generalised quadrangle of order $q^{2}$ with an antiregular base point is constructed, hence a Laguerre plane $\operatorname{LP}\left(q^{2}\right)$ of order $q^{2}$. If $q=3$, a unique Laguerre plane of order 9 exists by Steinke [15], and $\mathrm{Q}^{-}(3,3)$ is the unique BLT-set of the corresponding generalised quadrangle of order 9 .

Fix a BLT-set (of points) of $\mathrm{H}\left(3, q^{2}\right)$. As before it gives, by duality, a BLT-set $B$ of lines of $\mathrm{Q}^{-}(5, q)$, that is a set of mutually skew lines of $\mathrm{Q}^{-}(5, q)$ such that no three of them have a common transversal on $\mathrm{Q}^{-}(5, q)$. This is an egg $\mathrm{O}(2,2, q)$. The tangent space at the line $\ell$ of $\mathrm{O}(2,2, q)$ is the tangent space of $Q^{-}(5, q)$ at $\ell$. On the other hand, embed $\mathrm{Q}^{-}(5, q)$ in $\mathrm{Q}^{-}(7, q)$ as a section with a 5-dimensional space. Fix a point $P$ on $\mathrm{Q}^{-}(7, q) \backslash \mathrm{Q}^{-}(5, q)$ with $\mathrm{Q}^{-}(5, q)$ in $P^{\perp}$ and consider a $\mathrm{PG}(6, q)$ in $\mathrm{PG}(7, q)$ containing $\mathrm{Q}^{-}(5, q)$, but not $P$. The egg $\mathrm{O}(2,2, q)$ defines a translation generalised quadrangle of order $q^{2}$, which is isomorphic to the one constructed as in Theorem 3.2. Indeed, the isomorphism is the following. The special points correspond. For any line, say $\ell$, in the BLT-set, the plane $\langle P, \ell\rangle$ corresponds to $\ell$. Any line on $\langle P, \ell\rangle$ not containing $P$ has a polar 5-dimensional space (w.r.t. $\mathrm{Q}^{-}(7, q)$ ) which intersects $\mathrm{PG}(6, q)$ in a 4-dimensional space contaning the tangent space to $\mathrm{Q}^{-}(5, q)$ at $\ell$. For any plane, say $\pi$, of $\mathrm{Q}^{-}(7, q)$ which meets $P^{\perp}$ in a line not containing $P$ on some $\langle P, \ell\rangle$, the 3 -dimensional space $\langle P, \pi\rangle$ intersects $\mathrm{PG}(6, q)$ in a plane containing the relevant $\ell$. A point $P^{\prime}$ of $\mathrm{Q}^{-}(7, q)$ not in $P^{\perp}$ corresponds to the intersection of the line $P P^{\prime}$ with $\mathrm{PG}(6, q)$.

\section{BLT-sets in $T_{2}^{*}(\mathscr{H})$}

De Soete and Thas [4] study $\mathrm{T}_{2}^{*}(\mathscr{H})$. They show that the affine points of any affine line not meeting $\mathscr{H}$ are a BLT-set. Further, any two non-collinear points of $\mathrm{T}_{2}^{*}(\mathscr{H})$ 
are contained in a unique such BLT-set (they use this to give a characterisation of the underlying generalised quadrangle, with more assumptions). They determine all BLT-sets of $\mathrm{T}_{2}^{*}(\mathscr{H})$ in the case that $\mathscr{H}$ is regular. They give examples of BLT-sets in the dual of $\mathrm{T}_{2}^{*}(\mathscr{H})$.

\section{Partial BLT-sets of $Q(2 n, q), q$ odd, and partial flocks of cones}

O'Keefe and Thas in [9] have given a generalisation of the definition and of the main properties of flocks of quadratic cones of $\operatorname{PG}(3, q)$, hence of BLT-sets of Q $(4, q)$, to partial flocks of quadratic cones of PG $(2 n-1, q)$, hence of partial BLT-sets of $\mathrm{Q}(2 n, q)$. In $\mathrm{PG}(2 n-1, q)$, let $\mathscr{K}$ be the cone with vertex the point $v$ and base a parabolic quadric $\mathrm{Q}(2 n-2, q)$ in a hyperplane not containing $v$. A partial flock of $\mathscr{K}$ of size $k$ is a set of hyperplanes $\left\{\pi_{1}, \pi_{2}, \ldots, \pi_{k}\right\}$, each not on $v$, such that $\pi_{i} \cap \pi_{j}$ meets $\mathscr{K}$ in a non-singular elliptic quadric, for all $i \neq j, i, j \in\{1,2, \ldots, k\}$. Embed $\mathscr{K}$ in $\mathrm{Q}^{+}(2 n+1, q)$ as a section with a suitable $(2 n-1)$-dimensional space, and denote by $\perp$ the polarity defined by $\mathrm{Q}^{+}(2 n+1, q)$. Hence, each partial flock defines a partial ovoid of $\mathrm{Q}^{+}(2 n+1, q)$ of size $k q+1$ consisting of the $k$ conics $\pi_{i}^{\perp} \cap \mathrm{Q}^{+}(2 n+1, q)$, which are mutually tangent at a common point. On the other hand, for $q$ odd, embed $\mathscr{K}$ in $\mathrm{Q}(2 n, q)$ as a hyperplane section, and denote by $\perp^{\prime}$ the polarity defined by $\mathrm{Q}(2 n, q)$; for each $\pi_{i}$ in the partial flock, let $p_{i}$ the point of $\mathrm{Q}(2 n, q)$ defined by $\pi_{i}^{1^{\prime}} \cap \mathrm{Q}(2 n, q)=\left\{v, p_{i}\right\}$, and put $v=p_{0}$. The set $\left\{p_{0}, p_{1}, \ldots, p_{k}\right\}$ is a partial BLT-set of $\mathrm{Q}(2 n, q)$. For more details, see [9].

Let $q$ be odd. Following [2] and [9], let us write some examples of partial BLTsets of $\mathrm{Q}(6, q)$ arising from (possibly partial) BLT-sets of $\mathrm{Q}(4, q)$. Note that this extends also to $\mathrm{Q}(2 n, q)$ with similar argument. As a model for $\mathrm{Q}(6, q)$ take $x_{0} x_{1}+$ $x_{2} x_{3}-x_{4}^{2}+x_{5} x_{6}=0$, which contains the cone $\mathscr{K}: x_{0} x_{1}+x_{2} x_{3}-x_{4}^{2}=x_{6}=0$, having vertex $p_{0}=(0,0,0,0,0,1,0)$. Let $\mathscr{F}=\left\{\pi_{i}: i \in \mathscr{I}\right\}$ be a (partial) flock of $\mathscr{K}$. If $\pi_{i}$ has equation $a_{i} x_{0}+b_{i} x_{1}+c_{i} x_{2}+d_{i} x_{3}+e_{i} x_{4}+x_{5}=x_{6}=0$, then its polar line with respect to $\mathrm{Q}(6, q)$ intersects $\mathrm{Q}(6, q)$ at the further point $p_{i}=\left(b_{i}, a_{i}, d_{i}, c_{i},-e_{i} / 2, e_{i}^{2} / 4-a_{i} b_{i}-c_{i} d_{i}, 1\right)$ and the corresponding partial BLTset consists of $p_{0}$ and of the points $p_{i}$ 's.

Any (partial) BLT-set of $\mathrm{Q}(4, q): x_{0} x_{1}-x_{4}^{2}+x_{5} x_{6}=x_{2}=x_{3}=0$ is a partial BLT-set of $\mathrm{Q}(6, q)$, and any (partial) flock of $\mathscr{K}^{\prime}: x_{0} x_{1}-x_{4}^{2}=x_{2}=x_{3}=x_{6}=0$ canonically extends to a partial flock of $\mathscr{K}$ by reading the equations of the planes of the flock of $\mathscr{K}^{\prime}$ as equations of hyperplanes in $\operatorname{PG}(5, q): x_{6}=0$.

The linear flock of $\mathscr{K}^{\prime}$ defines a (partial) linear flock of $\mathscr{K}$ whose hyperplanes are $\pi_{t}: t x_{0}-\alpha t x_{1}+x_{5}=x_{6}=0, t \in \mathrm{GF}(q)$, where $\alpha$ is a fixed nonsquare in $\operatorname{GF}(q)$, the corresponding partial BLT-set is a conic and has $p_{t}=\left(-\alpha t, t, 0,0,0, \alpha t^{2}, 1\right)$. Any 
of these hyperplanes $\pi_{t}$ 's can be replaced by $\pi_{t}^{\prime}: 2 t x_{0}+t^{2} x_{2}-\alpha x_{3}+x_{5}=x_{6}=0$, as it can be easily checked that $\left(\mathscr{F}-\left\{\pi_{t}\right\}\right) \cup\left\{\pi_{t}^{\prime}\right\}$ is again a partial flock, so that $p_{t}$ is replaced by $p_{t}^{\prime}=\left(0,2 t,-\alpha, t^{2}, 0, \alpha t^{2}, 1\right)$, but only one such replacement can be done, as $\left\{p_{t}^{\prime}: t \in \mathrm{GF}(q)\right\}$ is contained in the plane $\Pi: x_{2}+\alpha x_{6}=x_{5}-\alpha x_{3}=x_{0}=x_{4}=0$ of $\mathrm{Q}(6, q)$. The partial ovoid of size $q^{2}+1$ of $\mathrm{Q}^{+}(7, q)$ corresponding to the original BLT-set is an elliptic quadric $\mathrm{Q}^{-}(3, q)$, and we can replace one conic on it with another one, which is tangent to all the other conics at $p_{0}$.

Any Kantor flock of $\mathscr{K}^{\prime}$ defines a (partial) flock of $\mathscr{K}$ whose hyperplanes are $\pi_{t}: t x_{0}-\alpha t^{\sigma} x_{1}+x_{5}=x_{6}=0, t \in \mathrm{GF}(q)$, where $\alpha$ is a fixed nonsquare in $\operatorname{GF}(q)$ and $\sigma \in A u t(\mathrm{GF}(q))$, the corresponding partial BLT-set being $p_{t}=$ $\left(-\alpha t^{\sigma}, t, 0,0,0, \alpha t^{\sigma+1}, 1\right)$. Here any of the $\pi_{t}$ 's can be replaced by $\pi_{t}^{\prime}: t x_{0}-$ $\alpha t^{\sigma} x_{1}+c x_{2}+d x_{3}+e x_{4}+x_{5}=x_{6}=0$, for $c, d, e \in \mathrm{GF}(q)$ such that $e^{2}=c d$, and $p_{t}$ is replaced by $p_{t}^{\prime}=\left(-\alpha t^{\sigma}, t, d, c, \sqrt{c d}, \alpha t^{\sigma+1}, 1\right)$. One can replace as many points $p_{t}$ 's as one likes, $\left\{p_{t}^{\prime}: t \in \mathrm{GF}(q)\right\}$ is contained in $x_{2} x_{3}-x_{4}^{2}=0$. The partial ovoid of size $q^{2}+1$ of $\mathrm{Q}^{+}(7, q)$ corresponding to the original BLT-set is contained in a 4-dimensional space, and we can replace any conic on it with another one, not in that 4-dimensional space, which is tangent to all the other conics at $p_{0}$.

Finally, any flock of $\mathscr{K}^{\prime}$ defines a (partial) flock of $\mathscr{K}$ whose hyperplanes are $\pi_{t}: a_{t} x_{0}+b_{t} x_{1}+c_{t} x_{4}+x_{5}=x_{6}=0, t \in \mathrm{GF}(q)$, for suitable $a_{t}, b_{t}, c_{t} \in \mathrm{GF}(q)$, the corresponding partial BLT-set being $p_{t}=\left(b_{t}, a_{t}, 0,0,-c_{t} / 2, c_{t}^{2} / 4-a_{t} b_{t}, 1\right)$. One can replace any of the $\pi_{t}$ 's by $\pi_{t}^{\prime}: a_{t} x_{0}+b_{t} x_{1}+d x_{2}+c_{t} x_{4}+x_{5}=x_{6}=0$, and $p_{t}$ is replaced by $p_{t}^{\prime}=\left(b_{t}, a_{t}, d, 0,-c_{t} / 2, c_{t}^{2} / 4-a_{t} b_{t}, 1\right)$, which lies on the line joining $p_{t}$ and $(0,0,1,0,0,0,0)$. The partial ovoid of size $q^{2}+1$ of $\mathrm{Q}^{+}(7, q)$ corresponding to the original BLT-set is contained in a 5-dimensional space, and we can replace one conic on it with another one, not in that 5-dimensional space, which is tangent to the other conics at $p_{0}$.

The size of a partial flock of a quadratic cone of $\operatorname{PG}(2 n-1, q), q$ even, is at most $q$ ([9, Theorem 2]), and this size actually occurs. On the other hand, for $q$ odd, examples of partial flocks of size $q$ of $\operatorname{PG}(2 n-1, q)$, hence of partial BLT-sets of $\mathrm{Q}(2 n, q), n \geq 2$, of size $q+1$, can be easily obtained as before, by taking a BLT-set of $\mathrm{Q}(4, q)$, but the theoretical bound for partial BLT-sets is $q^{(2 n-2) / 2}+1$ ([9, Theorem 6]), while the only known example before the results of this section of (partial) BLT-set of $\mathrm{Q}(2 n, q), n \geq 3$, having size greater than $q+1$ was for the particular case $\mathrm{Q}(6,3)$ and is of size 7 ([9, Section 6]).

Next, we will give examples of (partial) BLT-sets of $\mathrm{Q}(4 h, q), q$ odd, that have size greater than $q+1$. First, observe that the algebraic condition in Lemma 4.1 generalises immediately to quadrics $\mathrm{Q}(4 n, q)$ for $n \geq 2$ (and $q$ odd).

LEMMA 7.1. Let $q$ be odd. Let $\mathrm{Q}=\mathrm{Q}(2 n, q)$ and suppose $x, y, z$ are linearly independent vectors of $V$ such that $\langle x\rangle,\langle y\rangle,\langle z\rangle$ are singular points with respect to $Q$. 
Then $\langle x, y, z\rangle^{\perp} \cap \mathrm{Q}$ is an elliptic quadric if and only if $-2 f(x, y) f(y, z) f(z, x) / \Delta$ is a non-square in $\mathrm{GF}(q)$.

Proof. Mutatis mutandum, see proof of Lemma 4.1.

Then, following the ideas of Penttila [12], we can fix an alternative model for $\mathrm{Q}(4 n, q)$.

Let $q$ be any odd prime power, and put

$$
V=\left\{\left(x_{1}, \ldots, x_{n} ; y_{1}, \ldots, y_{n} ; a\right): x_{i}, y_{i} \in \mathrm{GF}\left(q^{2}\right), i=1, \ldots, n, a \in \mathrm{GF}(q)\right\} .
$$

The map $Q: V \mapsto \mathrm{GF}(q)$ defined as

$$
\begin{aligned}
& Q\left(x_{1}, x_{2}, \ldots, x_{n} ; y_{1}, y_{2}, \ldots, y_{n} ; a\right) \\
& \quad=N\left(x_{1}\right)+N\left(x_{2}\right)+\cdots+N\left(x_{n}\right)+N\left(y_{1}\right)+N\left(y_{2}\right)+\cdots+N\left(y_{n}\right)+a^{2},
\end{aligned}
$$

where $N(z)=z^{1+q}$ is the norm over $\operatorname{GF}(q)$, is a nondegenerate quadratic form over $V$ with discriminant $\Delta=2$. The associated bilinear polar form is

$$
\begin{aligned}
& f\left(\left(x_{1}, x_{2}, \ldots, x_{n} ; y_{1}, y_{2}, \ldots, y_{n} ; a\right),\left(x_{1}^{\prime}, x_{2}^{\prime}, \ldots, x_{n}^{\prime} ; y_{1}^{\prime}, y_{2}^{\prime}, \ldots, y_{n}^{\prime} ; a^{\prime}\right)\right) \\
&= T\left(x_{1} x_{1}^{\prime q}\right)+T\left(x_{2} x_{2}^{\prime q}\right)+\cdots+T\left(x_{n} x_{n}^{\prime q}\right)+T\left(y_{1} y_{1}^{\prime q}\right)+T\left(y_{2} y_{2}^{\prime q}\right) \\
&+\cdots+T\left(y_{n} y_{n}^{\prime q}\right)+2 a a^{\prime}
\end{aligned}
$$

where $T(z)=z+z^{q}$ is the trace over $\operatorname{GF}(q)$.

Using BLT-sets of $\mathrm{Q}(4, q)$ in this model, we construct partial BLT-sets of $\mathrm{Q}(4 n, q)$ of size $n(q+1)$.

THEOREM 7.2. Suppose $q$ is congruent to 1 or 3 modulo 8 . Let $B_{1}, \ldots, B_{k}$ be BLT-sets of the quadric $\mathrm{Q}(4, q)$ with equation $Q(x, y, a)=N(x)+N(y)+a^{2}=0$ with $x, y \in \operatorname{GF}\left(q^{2}\right)$ and $a \in \mathrm{GF}(q)$. Let $P_{1}, P_{2}, \ldots, P_{n} \in\left\{B_{1}, \ldots, B_{k}\right\}$. Then

$$
\begin{aligned}
\left\{\left(x_{1}, 0, \ldots, 0 ; y_{1}, 0, \ldots, 0 ; 1\right):\left(x_{1}, y_{1}, 1\right) \in P_{1}\right\} \\
\quad \cup\left\{\left(0, x_{2}, \ldots, 0 ; 0, y_{2}, \ldots, 0 ; 1\right):\left(x_{2}, y_{2}, 1\right) \in P_{2}\right\} \\
\quad \cup \cdots \cup\left\{\left(0,0, \ldots, x_{n} ; 0,0, \ldots, y_{n} ; 1\right):\left(x_{n}, y_{n}, 1\right) \in P_{n}\right\}
\end{aligned}
$$

is a partial BLT-set of $\mathrm{Q}(4 n, q)$.

Proof. The proof follows by Lemma 7.1, provided also -2 is a nonsquare in GF $(q)$, which is equivalent with $q$ congruent to 1 or 3 modulo 8 , when $n \geq 2$, because this is required when the points $\langle x\rangle,\langle y\rangle,\langle z\rangle$ belong to different $P_{i}$, hence they are such that $f(x, y) f(y, z) f(z, x)=-2$. 
Note that some BLT-sets of $\mathrm{Q}(4, q)$ have a nice representation in this model (Penttila [12]):

Classical BLT-set: $\left\{(x ; 0 ; 1): x \in \mathrm{GF}\left(q^{2}\right), N(x)=-1\right\}$;

Fisher BLT-set: $\left\{\left(b x^{2} ; 0 ; 1\right): x \in \mathrm{GF}\left(q^{2}\right), N(x)=1\right\} \cup\left\{\left(0 ; b y^{2} ; 1\right): y \in\right.$ $\mathrm{GF}\left(q^{2}\right), N(y)=1$ \} for $b$ a fixed element of $\mathrm{GF}\left(q^{2}\right)$ such that $N(b)=-1$;

Fisher-Thas-Walker BLT-set: $\left\{\left(a x ; b x^{2} ; 1\right): x \in \mathrm{GF}\left(q^{2}\right), N(x)=1\right\}$ where $q$ is congruent to 2 modulo 3, $a, b \in \mathrm{GF}(q)$ such that $N(a)=-4 / 3$ and $N(b)=1 / 3$;

Mondello BLT-set: $\left\{\left(a x^{2} ; b x^{3} ; 1\right): x \in \mathrm{GF}\left(q^{2}\right), N(x)=1\right\}$ where $q$ is congruent to 1 or 9 modulo $10, a, b \in \mathrm{GF}(q)$ such that $N(a)=-4 / 5$ and $N(b)=-1 / 5$.

It is clear that any other BLT-set of $\mathrm{Q}(4, q)$, once written in a suitable way in the model of [12], might be used for an explicit construction.

Note that $\mathrm{Q}(4 n, q)$ is embedded in $\mathrm{Q}(4 n+2, q)$, hence any partial BLT-set of $\mathrm{Q}(4 n, q)$ is also a partial BLT-set of $\mathrm{Q}(4 n+2, q)$.

The partial ovoid of $\mathrm{Q}^{+}(4 n+1, q)$ corresponding to the partial flock and the partial BLT-set (see [9, Section 4.2]) is the union of the ovoids of $\mathrm{Q}^{+}(5, q)$ associated with any of the BLT-sets, is of size $n q^{2}+1$ and consists of $n q$ conics mutually tangent at a common point. If $P_{i}$ is classical for all $i=1,2, \ldots, n$, then the conics cover $n$ elliptic quadrics $\mathrm{Q}^{-}(3, q)$.

The preceding procedure obtained partial ovoids of $\mathrm{Q}^{+}(4 n+1, q)$ from ovoids of $\mathrm{Q}^{+}(5, q)$. A related construction is the following, which holds also when $q$ is even.

Let $V$ be a vector space with a quadratic form with associated nondegenerate bilinear form $f$ and suppose that $V$ is an orthogonal direct sum, say $V=\langle v\rangle \perp U_{1} \perp \cdots \perp U_{h}$.

THEOREM 7.3. Let $\mathscr{O}_{i}$ be a partial ovoid of $\left\langle v, U_{i}\right\rangle$ containing $\langle v\rangle$ and disjoint from $U_{i}$. Then $\mathscr{O}=\mathscr{O}_{1} \cup \cdots \cup O_{h}$ is a partial ovoid of the quadric arising from the quadratic form.

Proof. Let $P, Q$ be distinct points of $\mathscr{O}$. Hence $P \in \mathscr{O}_{i}$ and $Q \in \mathscr{O}_{j}$ for some $i, j$. If $i=j$, since $\mathscr{O}_{i}$ is a partial ovoid, $P$ and $Q$ are not collinear. Suppose $i \neq j$. As $P \neq\langle v\rangle, P$ is not collinear with $\langle v\rangle$. Now, $P^{\perp}$ contains $U_{j}$, so $P^{\perp} \cap\left\langle v, U_{j}\right\rangle=U_{j}$, and $Q \notin U_{j}$. Hence $P$ and $Q$ are not collinear.

REMARK. Note we here are not assuming $\operatorname{dim}\left(U_{i}\right)=\operatorname{dim}\left(U_{j}\right)$.

Let us now generalise the example of $[9$, Section 6$]$.

THEOREM 7.4. Let $p$ be a prime congruent to 1 or 3 modulo 8 , and let $n$ be a ositive integer. There exists a partial flock of size $2 p n$ of the cone of $\mathrm{PG}(2 p n-1, p)$ vith vertex a point and basis a parabolic quadric, with the corresponding partial $3 \mathrm{LT}$-set of $\mathrm{Q}(2 p n, p)$ admitting the symmetric group of degree $2 p n+1$. 
PROOF. In $\mathrm{PG}(2 p n, p)$, fix the quadric $\mathrm{Q}=\mathrm{Q}(2 p n, p)$ with equation $x_{0}^{2}+$ $x_{1}^{2}+\cdots+x_{2 p n}^{2}=0$, with associated form $f$. For all $i=0,1, \ldots, 2 p n$, put $A_{i}=\left(y_{0}, y_{1}, \ldots, y_{2 p n}\right)$ with $y_{i}=0$ and $y_{j}=1$ for $j \neq i$. Hence $A_{i} \in \mathrm{Q}$ and $\operatorname{disc}(\mathrm{Q})=2^{1+2 p n}$, then $\operatorname{disc}(\mathrm{Q})$ is congruent to $2^{1+2 n}$ modulo $p$, which is a nonsquare by hypothesis. Also, for any $i \neq j$ we have $f\left(A_{i}, A_{j}\right)=2(p n-1)=-2$. This implies that $-2 f\left(A_{i}, A_{j}\right) f\left(A_{i}, A_{h}\right) f\left(A_{h}, A_{j}\right)$ is a square, and by Lemma 7.1 this is a partial BLT-set. Moreover, this is contained in the hyperplane $(1,1, \ldots, 1)^{\perp}$, which intersects $\mathrm{Q}$ in a $\mathrm{Q}^{-}(2 p n-1, p)$.

Finally, using the software MAGMA [3], we have constructed partial BLT-sets of size $2 q+1$ of the quadric $\mathscr{Q}(6, q)$ for $q=5,7,9$. Can these be generalised? Is this the maximum size for partial BLT-sets of $\mathscr{Q}(6, q)$ ?

\section{Aknowledgements}

This work was done partly while the first author was a Visiting Professor at the University of Adelaide and at the University of Western Australia, partly while the second author was a C.N.R. Visiting Professor at the Università di Roma 'Tor Vergata', partly while the third author was a C.N.R. Visiting Professor at the Università di Roma 'La Sapienza' and at the Università di Napoli, and was also supported by the Australian Research Council.

\section{References}

[1] L. Bader, G. Lunardon and I. Pinneri, 'A new semifield flock', J. Comb. Theory Ser. (A) 86 (1999), $49-62$.

[2] L. Bader, G. Lunardon and J. A. Thas, 'Derivation of flocks of quadratic cones', Forum Math. 2 (1990), 163-174.

[3] J. Cannon and C. Playoust, 'An introduction to MAGMA', Technical report (University of Sydney, Australia, 1993).

[4] M. De Soete and J. A. Thas, 'A characterization theorem for the generalized quadrangle $\mathrm{T}_{2}^{*}(\mathscr{O})$ of order $(s, s+2)$ ', Ars Combin. 17 (1984), 225-242.

[5] A. Delandtsheer, 'Dimensional linear spaces', in: Handbook of incidence geometry (ed. F. Buekenhout) (North-Holland, 1995).

[6] N. L. Johnson, 'Derivation of partial flocks of quadratic cones', Rend. Mat. Serie VII 12 (1992), 817-848.

[7] W. M. Kantor, 'Note on generalized quadrangles, flocks and BLT-sets', J. Combin. Theory Ser. (A) 58 (1991), 153-157.

[8] N. Knarr, 'A geometric construction of generalized quadrangles from polar spaces of rank three', Resultate Math. 21 (1992), 332-344.

[9] C. M. O'Keefe and J. A. Thas, 'Partial flocks of quadratic cones with a point vertex in $\operatorname{PG}(n, q)$, $n$ odd', J. Algebraic Combin. 6 (1997), 377-392. 
[10] S. E. Payne and J. A. Thas, Finite generalized quadrangles (Pitman, London, 1984).

[11] —, 'Conical flocks, partial flocks, derivation, and generalized quadrangles', Geom. Dedicata 38 (1991), 229-243.

[12] T. Penttila, 'Regular cyclic BLT-sets', Rend. Circ. Mat. Palermo, supplemento Ser. II 53 (1998), 167-172.

[13] J. J. Seidel, 'A survey on two-graphs', in: Teorie Combinatorie (ed. B. Segre) (Accad. Naz. Lincei, Roma, 1976).

[14] E. E. Shult and J. A. Thas, 'Construction of polygons from buildings', Proc. London Math. Soc. 71 (1995), 397-440.

[15] G. F. Steinke, 'A remark on Benz planes of order 9', Ars Combin. 34 (1992), 257-267.

[16] J. A. Thas, 'Generalized quadrangles and flocks of cones', European J. Comb. 8 (1987), 441-452.

[17] — 'Generalized polygons', in: Handbook of incidence geometry (ed. F. Buekenhout) (NorthHolland, 1995).

Dipartimento di Matematica e Applicazioni

CSIRO ICT Centre

Università di Napoli 'Federico II'

GPO Box 664

Complesso di Monte S. Angelo

Canberra 2601

Via Cintia - Edificio T

Australia

I-80126 Napoli

e-mail: christine.okeefe@csiro.au

Italy

e-mail: laura.bader@dma.unina.it

School of Mathematics and Statistics (M019)

The University of Western Australia

35 Stirling Highway

Crawley 6009 WA

Australia

e-mail: penttila@maths.uwa.edu.au 
J. Aust. Math. Soc. 76 (2004) 